\title{
Richtiger Knotenabstand beim Bauchverschluss
}

\section{Zur Prävention von Narbenhernien nach Laparotomie weist ein enger Knotenabstand beim Verschluss der Faszie deutliche Vorteile auf.}

\begin{abstract}
Die Inzidenz von Narbenhernien nach Laparotomien bewegt sich in einer Größenordnung zwischen 10\% und 23\% und erreicht bei speziellen Risikogruppen Werte bis $38 \%$. In den USA werden bei 4-5 Millionen Laparotomien pro Jahr etwa 348.000 Operationen wegen Narbenhernien durchgeführt. Es lohnt also jede Anstrengung, ein derartiges Ereignis zu vermeiden.
\end{abstract}

In einer prospektiven, randomisierten und kontrollierten Studie in zehn Krankenhäusern wurde zwischen 2009 und 2012 in den Niederlanden die Fasziennaht nach Mittellinien-Laparotomie untersucht. Bei 284 Patienten wurden Abstände von $1 \mathrm{~cm}$ zwischen den Knoten eingehalten, bei 276 Patienten nur $5 \mathrm{~mm} .97 \%$ der Patienten beendeten das Follow-up.

Bei den Patienten mit engem Knotenabstand betrug die mediane Anzahl von Stichen $45 \pm 12 \mathrm{im}$ Vergleich zu $25 \pm 10$ bei weitem Abstand. Der Quotient aus Nahtlänge und Wundlänge war in der Gruppe der dichter gesetzten Knoten höher $(5,0 \pm 1,5$ vs. $4,3 \pm 1,4)$.

Naturgemäß erforderte der engmaschigere Faszienverschluss mehr Zeit (14 \pm 6 vs. $10 \pm 4 \mathrm{~min})$. Doch diese Mühe lohnte sich: Nach einem Jahr wiesen $21 \%$ der Patienten mit weitem Abstand eine Narbenhernie auf, aber nur 13\% der Patienten mit engerem Abstand. Die Rate perioperativer Komplikationen, insbesondere auch postoperativer Schmerzen, war in beiden Gruppen gleich.

- Deerenberg EB et al. Small bites versus large bites for closure of abdominal midline incisions (STICH): a double-blind, multicentre, randomised controlled trial. Lancet. 2015;386:1254-60

\section{KOMMENTAR}

Was einen an der Studie am meisten erstaunt, ist der Umstand, dass sie erst jetzt durchgeführt wurde. Dies gilt umso mehr, wenn man bedenkt, dass Narbenhernien

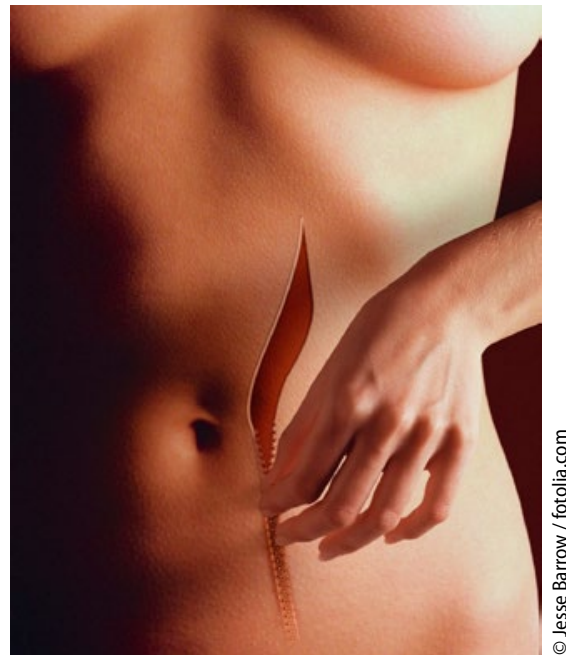

nach Laparotomien ein häufiges Phänomen sind, welches offensichtlich von den Chirurgen kaum wahrgenommen wird - zumindest nicht von denen, die die primäre Operation durchgeführt haben. Von großen Chirurgen wird der Wundverschluss ja gern auch an nachgeordnete Mitarbeiter delegiert, wodurch das Problem weiter aus dem Blickfeld gerät. Wenn aber ein derart einfaches Vorgehen wie die Verdopplung der Zahl der Stiche beim Faszienverschluss die Rate an Narbenhernien fast halbieren kann, so dürften die paar Minuten verlängerte Operationsdauer wirklich nicht ins Gewicht fallen.

Prof. Dr. med. H. S. FüeßI

\section{Hier steht eine Anzeige.}

